\title{
Fitting Cure Rate Model to Breast Cancer Data of Cancer Research Center
}

\author{
Ahmad Reza Baghestani' ${ }^{2}$, Farid Zayeri², Mohammad Esmaeil Akbari ${ }^{1}$, Leyla \\ Shojaee $^{1}$, Naghmeh Khadembashi ${ }^{4}$, Parviz Shahmirzalou ${ }^{1,2,3, *}$
}

\begin{abstract}
Background: The Cox PH model is one of the most significant statistical models in studying survival of patients. But, in the case of patients with long-term survival, it may not be the most appropriate. In such cases, a cure rate model seems more suitable. The purpose of this study was to determine clinical factors associated with cure rate of patients with breast cancer. Materials and Methods: In order to find factors affecting cure rate (response), a non-mixed cure rate model with negative binomial distribution for latent variable was used. Variables selected were recurrence cancer, status for HER2, estrogen receptor (ER) and progesterone receptor (PR), size of tumor, grade of cancer, stage of cancer, type of surgery, age at the diagnosis time and number of removed positive lymph nodes. All analyses were performed using PROC MCMC processes in the SAS 9.2 program. Results: The mean (SD) age of patients was equal to 48.9 (11.1) months. For these patients, 1,5 and 10-year survival rates were 95,79 and 50 percent respectively. All of the mentioned variables were effective in cure fraction. Kaplan-Meier curve showed cure model's use competence. Conclusions: Unlike other variables, existence of ER and PR positivity will increase probability of cure in patients. In the present study, Weibull distribution was used for the purpose of analysing survival times. Model fitness with other distributions such as $\log -\mathrm{N}$ and log-logistic and other distributions for latent variable is recommended.
\end{abstract}

Keywords: Breast neoplasms - cure fraction - cure rate model - negative binomial distribution - survival analysis

Asian Pac J Cancer Prev, 16 (17), 7923-7927

\section{Introduction}

Breast cancer $(\mathrm{BC})$ is the most common cancer among women worldwide (Lotfnezhad et al., 2015). In 2012, there were 1.67 million incident cases from 140 of 184 countries. Around $39 \%$ of all cases in 2012 were from Asian countries (Kim et al., 2015). Unfortunately, BC incidence is rising among Iranian women. That includes $16 \%$ and $22.9 \%$ of all and invasive cancers respectively (Veisy et al., 2015). Incidence rate of BC in Isfahan province of Iran rates to 30 per 100,000 population (Movahedi et al., 2012; Baghestani et al., 2015). Taghavi et al states that there is increasing trend of $\mathrm{BC}$ in Iran, although mortality rate of $\mathrm{BC}$ in Iran proves to be lower than in western countries (Taghavi et al., 2012).

Another study in patients with more than 9 involved lymph nodes, manifested that young age and estrogen tumor positivity was associated with improved outcomes. Race of patients was significant at 0.1 levels (Zaichner et al., 2014). Some researchers also surveyed the effect of fat diet and obesity on BC patient's survival (Kaviani et al., 2013; Xing et al., 2014).

Survival analysis is a collection of statistical procedures in which the outcome of interest is time until an event occurs. Time can be weeks, months or years; outcome can be death, disease, incidence or relapse from remission, recovery and so on (Kleinbaum et al., 2012). The purpose of most studies is to identify effective factors on survival of patients. In fact for the purpose of monitoring the effectiveness of cancer treatment and identifying prognostic factors of the disease, studies of cancer patient survival are required (Rahimzadeh et al., 2014). Some studies surveyed role of age, age at menarche, total reproductive age, age at first child birth, and menopausal status (Akhtar et al., 2015). Some other study surveyed role of obesity, alcohol consumption, inactivity, and hormone replacement therapy (Colditz et al., 2014; Howell et al., 2014). The Cox Proportional Hazards (PH) model is the most popular, semi-parametric model for the analysis of survival data (Kleinbaum et al., 2012). It assumes that all patients will eventually experience the main outcome (relapse or death). Therefore, Cox PH model cannot be used to identify prognostic factors associated with the cure procedure (Asano et al., 2013). In some circumstances, despite the fact that the follow-up period is long, some patients will not experience main event; hence, the

${ }^{1}$ Cancer Research Center, Shahid Beheshti University of Medical sciences, Tehran, ${ }^{2}$ Department of Biostatistics, ${ }^{3}$ Committee Research, ${ }^{4}$ Department of Englishsh language, School of Allied Medical Sciences, Shahid Beheshti University of Medical sciences, Tehran, Iran*For correspondence: pshkhoei@gmail.com 
censoring rate increases, leading to the overestimation of the survival rate (Mirzaei et al., 2014). Cure rate models can be used to investigate the heterogeneity between patients with cancer who are long-term survivors and those who are not. Kaplan-Meier curve can be used to identify long-term survivors. Survival curve has a plateau among long-term survivors. Then cure rate model may be useful to analyze data (Othus et al., 2012).

The aim of this study was to use cure rate model to determine the prognostic factors on survival of $\mathrm{BC}$ patients.

\section{Materials and Methods}

The present study is a prospective cohort study on 557 patients with $\mathrm{BC}$ who were admitted at Cancer Research Center (CCC) of Shahid Beheshti University of Medical Sciences, Tehran Iran, during 1994 to 2013. All patients went under Mastectomy Modified Radical (MRM) or Breast Conserving Surgery (BCS). Some of patients experienced the recurrence of the disease. In some cases, the patients or patient's family members were contacted to confirm if the patients were still alive. Variables recurrence cancer, status of and Human Epidermal growth factor Receptor-2 (HER2), Estrogen Receptor (ER), Progesterone Receptor (PR), size of tumor, grade of cancer, stage of cancer, type of surgery, age at the diagnosis time and Number of Removed Positive Lymph Nodes (NRPLN) were selected. Stage and grade of cancer were categorized to 3 and 4 degrees respectively. Size of tumor was categorized to 3 sizes (less than 2, 2-5 and more than $5 \mathrm{~cm}$ ).

BC seemed be more aggressive in younger patients; Consequently age at the diagnosis time was categorized to 4 age groups (less than 40,40 to 50,50 to 60 and older than 60) and NRPLN to 3 groups (zero NRPLN, between 1 to 9 and more than 10). For data analysis, Non-Mixed cure rate model with negative binomial distribution was employed. Weibull and uniform distribution were proposed for survival time and prior distribution of all

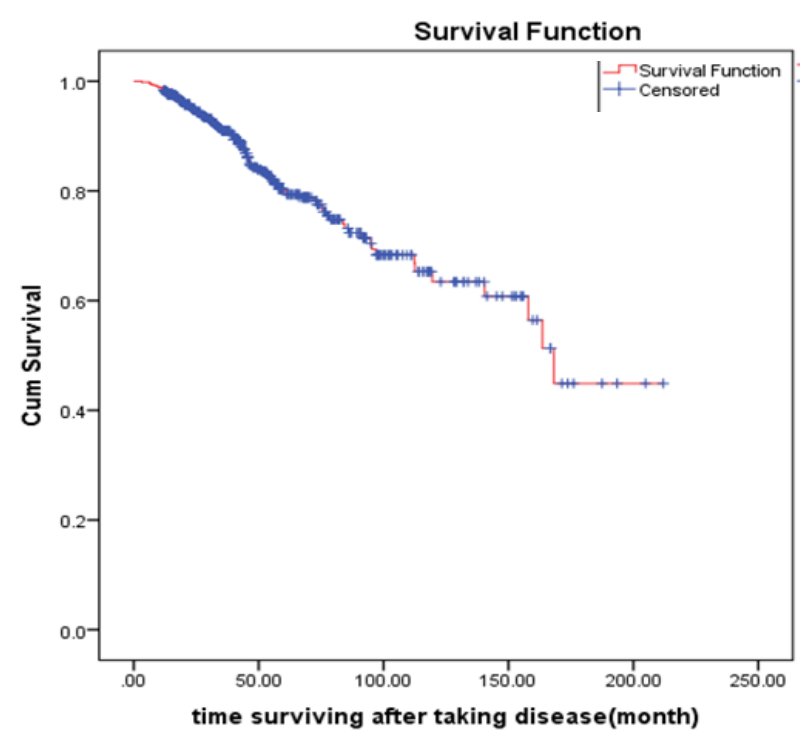

Figure 1. Kaplan-Meier Curve for Overall Cumulative Survival of Women with BC parameters in the model respectively. To identify the significant prognostic factors, credible intervals were used. Credible interval is a Bayesian form of confidence interval in domain of posterior probability distribution.

In cure rate models, covariates depend on $\theta$ through the following relationship: $\theta=\exp \left(x^{\prime} \beta\right)$. where $x$ is a $p^{*} 1$ vector of covariates and $\beta$ is a $p^{*} 1$ vector of regression coefficients. Using, $\theta=\exp \left(x^{\prime} \beta\right)$ in $P_{0}=\{1+\eta \theta\}^{-1 / \eta}$ the role of the regression coefficients for the cured and noncured group can be interpreted. Since $\eta>0$, the relation between the covariates and the cure rate is inverse (Cancho et al., 2015). For instance, if a covariates has a positive coefficient, an increase in its value implies a reduction in the cure rate (where all other covariates are kept constant). All analyses were carried out using PROC MCMC in SAS version 9.2 and the parameters were estimated through a Bayesian approach.

\section{Results}

Data from 557 female patients with breast cancer were analyzed. The mean (SD) age was equal to 48.86 (11.31).

Table 1. Baseline Characteristics of the Patients

\begin{tabular}{|c|c|c|c|}
\hline Variable & Category & No $(\%)$ & $\begin{array}{l}\text { Survival mean in month } \\
\text { (percent of death) }\end{array}$ \\
\hline \multicolumn{4}{|c|}{ Recurrence of cancer } \\
\hline & Yes & $96(17.2)$ & $56.85(65.6)$ \\
\hline & No & $461(82.8)$ & $49.70(6.3)$ \\
\hline \multicolumn{4}{|c|}{ Type of surgery } \\
\hline & $\mathrm{BCS}$ & $348(62.5)$ & $43.46(8)$ \\
\hline & MRM & $209(37.5)$ & $63.38(30.6)$ \\
\hline \multicolumn{4}{|l|}{ ER } \\
\hline & Yes & $388(69.7)$ & $49.75(13.4)$ \\
\hline & No & $169(30.3)$ & $53.65(23.7)$ \\
\hline \multicolumn{4}{|l|}{ PR } \\
\hline & Yes & $369(66.2)$ & $49.95(13.6)$ \\
\hline & No & $188(33.8)$ & $52.86(22.3)$ \\
\hline \multicolumn{4}{|l|}{ HER2 } \\
\hline & Yes & $127(22.8)$ & $57.93(29.9)$ \\
\hline & No & $430(77.2)$ & $48.86(12.6)$ \\
\hline \multicolumn{4}{|l|}{ Age } \\
\hline & Less than 40 & $120(21.5)$ & $52.69(20)$ \\
\hline & $40-50$ & $210(37.7)$ & $53.37(13.8)$ \\
\hline & $50-60$ & $137(24.6)$ & $50.25(13.1)$ \\
\hline & More than 60 & $90(16.2)$ & $43.93(23.3)$ \\
\hline \multicolumn{4}{|c|}{ tumor size $(\mathrm{cm})$} \\
\hline & less than 2 & $190(34.1)$ & 49.81(7.9) \\
\hline & $2-5$ & $291(52.2)$ & $52.65(16.2)$ \\
\hline & More than 5 & $76(13.6)$ & $47.17(39.5)$ \\
\hline \multicolumn{4}{|c|}{ NRPLN } \\
\hline & 0 & $268(48.1)$ & $56.10(6)$ \\
\hline & $0-9$ & $241(43.3)$ & $46.51(22)$ \\
\hline & 10 and More & $48(8.6)$ & $44.32(47.9)$ \\
\hline \multicolumn{4}{|c|}{ stage of cancer } \\
\hline & 1 & $126(22.6)$ & $52.05(5.6)$ \\
\hline & 2 & $252(45.2)$ & $52.69(8.3)$ \\
\hline & 3 & $163(29.3)$ & $48.51(32.5)$ \\
\hline & 4 & $16(2.9)$ & $39.09(68.8)$ \\
\hline \multicolumn{4}{|c|}{ Grade of cancer } \\
\hline & 1 & $61(11)$ & $53.09(6.6)$ \\
\hline & 2 & $312(56)$ & $51.69(12.5)$ \\
\hline & 3 & 184(33) & $48.94(26.6)$ \\
\hline
\end{tabular}


Fitting Cure Rate Model to Breast Cancer Data of Cancer Research Center

and Minimum and maximum follow-up times of patients were equal to 3.23 and 211.97 months, respectively, with the average of 50.93 and the median of 39.96 months. Among the patients, 92 of the patients died from breast cancer and the rest survived. Other descriptive statistics are shown in Table 1.

For these patients 1, 5 and 10-years survival rate was 95, 79 and 50 percent, respectively. Since cured models are appropriate for data with cured fraction, the KaplanMeier curve showed cured patients in the data (Figure 1).

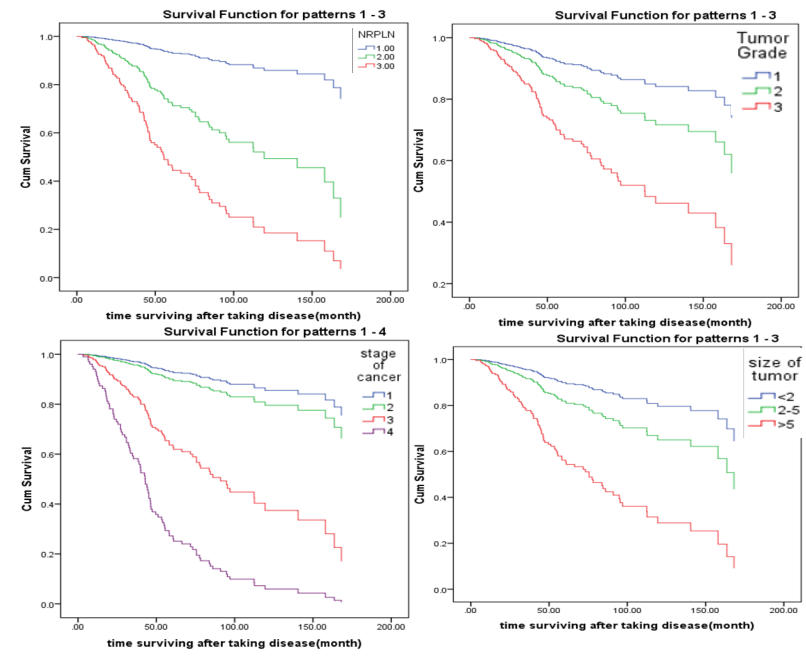

Figure 2. Kaplan-Meier Analysis Showing Disease-free Survival for Variables Stage and Grade of Cancer, Size of Tumor, NRPLN
Because of Fiqure1, the above mentioned variables were entered in Non-Mixed cure rate model with negative binomial distribution for the purpose of determining factors associated with cure rate of patients (Table 2).

These results manifested that all of the variables were significant on the cure rate of patients (Table 2). Sign in the mean of parameters shows role of variable in increase or decrease cure rate. For instance, positive sign causes a decrease in the cure rate. Kaplan-Meier analysis showed disease-free survival for variables such as stage of cancer, Tumor grade, size of tumor, NRPLN and age at the time of diagnosis (Figure 2).

There are sensible difference between the categories stage of cancer, Tumor grade, NRPLN and size of tumor while at the age group of 40-50 and 50-60 an overlap in the graph was observed. Fourth column in Table 2 shows cure rate. For instance, probability of cure in patients without recurrence of disease was $30 \%$ more than patients with recurrence of $\mathrm{BC}$ while probability of cure in patients with ER was $8 \%$ more than patients without ER.

\section{Discussion}

$\mathrm{BC}$ is the most common cancer among Iranian women. Incidence rate of BC was 30 cases per 100,000 Iranian patients (Movahedi et al., 2012). It is essential that mortality and survival of disease be improved by an early diagnosis. In some forms of cancers, such as BC, we expect to see long-term survival by early diagnosis (Najafi et al., 2013).

Table 2. Promotion Time Parameters of the Negative binomial model

\begin{tabular}{|c|c|c|c|c|c|c|}
\hline \multirow[t]{2}{*}{ Parameters } & \multirow[t]{2}{*}{ Category } & \multirow[t]{2}{*}{ Mean (SD) } & \multicolumn{2}{|c|}{ Credible Interval } & \multirow[t]{2}{*}{ Sig. } & \multirow[t]{2}{*}{ Cure rate } \\
\hline & & & $2.50 \%=$ & $97.50 \%$ & & \\
\hline \multirow[t]{2}{*}{ Recurrence of cancer } & Yes & $0.83(0.01)$ & 0.81 & 0.84 & $*$ & 0.71 \\
\hline & No & REF & - & - & - & - \\
\hline \multirow[t]{2}{*}{ Type of surgery } & MRM & $0.68(0.02)$ & 0.65 & 0.72 & $*$ & 0.77 \\
\hline & BCS & REF & - & - & - & - \\
\hline \multirow[t]{2}{*}{ ER } & Yes & $-0.29(0.01)$ & -0.31 & -0.27 & $*$ & 1.08 \\
\hline & No & REF & - & - & - & - \\
\hline \multirow[t]{2}{*}{ PR } & Yes & $-0.59(0.01)$ & -0.61 & -0.55 & $*$ & 1.16 \\
\hline & No & REF & & - & - & - \\
\hline \multirow[t]{2}{*}{ HER2 } & Yes & $0.89(0.01)$ & 0.87 & 0.92 & $*$ & 0.69 \\
\hline & No & REF & - & - & - & - \\
\hline \multirow[t]{4}{*}{ Age } & Less than 40 & REF & - & - & - & - \\
\hline & $40-50$ & $0.21(0.01)$ & 0.19 & 0.24 & $*$ & 0.93 \\
\hline & $50-60$ & $0.60(0.03)$ & 0.55 & 0.65 & $*$ & 0.79 \\
\hline & More than 60 & $0.46(0.01)$ & 0.43 & 0.49 & $*$ & 0.84 \\
\hline \multirow[t]{3}{*}{ tumor size $(\mathrm{cm})$} & less than 2 & $\mathrm{REF}$ & - & - & - & - \\
\hline & $2-5$ & $0.69(0.01)$ & 0.67 & 0.71 & $*$ & 0.76 \\
\hline & More than 5 & $-0.42(0.01)$ & -0.44 & -0.4 & $*$ & 1.12 \\
\hline \multirow[t]{3}{*}{ NRPLN } & 0 & REF & & - & - & - \\
\hline & $0-9$ & $0.24(0.01)$ & 0.22 & 0.27 & $*$ & 0.91 \\
\hline & 10 and More & $-0.85(0.02)$ & -0.89 & -0.81 & $*$ & 1.21 \\
\hline \multirow[t]{4}{*}{ stage of cancer } & 1 & REF & - & - & - & - \\
\hline & 2 & $0.21(0.01)$ & 0.2 & 0.23 & $*$ & 0.92 \\
\hline & 3 & $-0.44(0.01)$ & -0.46 & -0.42 & $*$ & 1.12 \\
\hline & 4 & $-0.59(0.01)$ & -0.6 & -0.57 & $*$ & 1.16 \\
\hline \multirow[t]{3}{*}{ Grade of cancer } & 1 & REF & - & - & - & - \\
\hline & 2 & $0.52(0.01)$ & 0.49 & 0.54 & $*$ & 0.82 \\
\hline & 3 & $1.05(0.01)$ & 1.03 & 1.08 & $*$ & 0.63 \\
\hline
\end{tabular}


Cure rate models are appropriate for determining effective factors on patient's survival and cure rate. Promotion time cure model was introduced first by Yakovlev and Tsodikov (1993). It was extended by Chen (1999) afterwards. In survival data analysis, we are facing a high percentage of individuals who will not experience the given event. These individuals are referred to as cured. There exists many cured individual in cancers like BC. Although it is more 20 years that cure rate models are introduced, they have not been addressed properly yet (Othus et al., 2012). These models are useful and effective, despite the fact that there are no cured patients. Under such conditions, these models will play the role of survival models appropriately.

In this study 1, 5 and 10-years survival rate was 95, 79 and 50 percent, respectively. Abdollahi et al estimated 5 -years survival of patients equal to $49 \%$. That is much lower than other studies estimations (Abdullah et al., 2013). On the other hand, Ziaie et al estimated 1, 5 and 10 -years patients survival 96,81 and $76 \%$, respectively that is more than the present study's estimations (Ziaei et al., 2013).

All Variables were diagnosed as factors contributing to Iranian women cure with BC. Recurrence of BC occurred in 96 (\%17) patients. Mean of survival time in patients with recurrence of $\mathrm{BC}$ have 7 month more than others. They also had 60 percent more death rate, comparatively speaking. Thus, recurrence of BC caused a decrease in the cure rate.

Many breast cancer patients normally select one of the two surgeries of MRM or BCS during their treatment period. In such cases examining patient's survival status and determining multi-year survival with surgical separation proves to be quite important. One study stated that patients who are undergoing BCS surgery have better performance and survival status than MRM ones(Acil et al., 2014). Patients with surgery of MRM had 20 month more survival, but near 23 percent more death rate than BCS ones. Another study showed that the long-term survival rate among women who undergo breast-conserving surgery is the same as those women who undergo radical mastectomy (Veronesi et al., 2002).

One important aspect of the role of pathology in the evaluation of $\mathrm{BC}$ is biomarker testing, as ER, PR and HER2. Biomarkers might be prognostics, predictive and both. Thus, it proves to be effective on treatment and survival of BC. This study proved that ER and PR inverse HER 2 caused an increase in the cure fraction. One study was performed in order to ascertain the role of receptors (ER, PR and HER2) in four groups (luminal A and B, HER 2 type and triple negative) with survival of patients with spinal bone metastases. The study showed that a survival time wasn't significant between groups; and yet patients with triple negative groups had shorter survival rate than luminal B (Bollen et al., 2015).

Rahimzadeh et al. in a study conducted on Iranian women patients with breast cancer, considered latent variable distribution (the number of remaining cancer cells in cancerous tissue after receiving treatment) as Poisson and negative binomial. In that study, patient's age was significant only in a model with negative binomial distribution. DIC criterion comparison of the two models revealed that this criterion was the least in a model with negative binomial distribution (Rahimzadeh et al., 2014). Another study that focused on age at the diagnosis time, showed age as an important factor in survival of patients with BC (Asif et al., 2014). Age at the diagnosis time was categorized into four groups in this study (less than 40 , 40 to 50,50 to 60 , more than 60 ). Third and fourth age group had less survival mean compared to those in the first group. This difference was sensible in the fourth group.

Tumor size is related to BC recurrence and survival time. More study showed a reverse relationship between tumor size and BC survival. The present study proves that, with increasing tumor size, percentage of death (7.9, 16.2 and $39.5 \%$ ) increase which proves to be a significant factor in cure rate. However, there is a heterogeneity in significance between categories of tumor size (Table 2). One study indicated that a decline of 1 and $1.5 \mathrm{~cm}$ in size of tumor was associated with a $10.3 \%$ and $23 \%$ reduction in mortality rate respectively (Narod et al., 2014). Another study showed a significant effect between tumor size and some other variables with overall survival of BC patients in Pakistan (Mahmood et al., 2015).

Lymph node involvement is a very important factor for patients with breast cancer (Orang et al., 2013), affecting the patient's survival (Chen et al., 2014). Patients with more than 10 NRPLN had 12 month less survival time and more than $42 \%$ more probability of dying from BC compared to patients with less than 10 NRPLN.

Cancer stage is one of the variables that affect the survival of patients. According to one study, 5 and 10 years survival rate of patients for all stages was 64.2 and 52.6 respectively (Rama et al., 2010). In the present study, the values of $93,91,59$ and 19 percent were estimated respectively.

Tumor grade is a description of normality status of cancer cells and therefore is an indicator of the ability of a tumor to grow and spread. The patients with tumor grade 1 (53.09) had better survival than patients with tumor grade 2 (51.69) and 3 (48.94). While, patients with tumor grade 3 had $20 \%$ more death than patients in grade 1 . This relationship was highly significant $(\mathrm{p}<0.001)$ in some studies (Sipetic-Grujicic et al., 2014).

$\mathrm{BC}$ is the most common cancer in women worldwide. Recognizing factors affecting cure rate of patients can be a significant factor in reducing death rate. There are limited studies on BC survival, which employed cure rate model to identify the clinical factors associated with cure (12). These cure rate models are introduced to explain the remaining cancer cells after treatment, using latent variable. Although them would be beneficial, even though there are no cured patients. Analysis showed that biologic variables (ER, PR and HER2) and recurrence of cancer, size of tumor, grade of cancer, stage of cancer, type of surgery, age at diagnosis and NRPLN were important on cure rate of $\mathrm{BC}$. Meanwhile, there were heterogeneity in sign in mean of tumor size, NRPLN and stage of cancer, maybe for correlation between these with other variables.

\section{Acknowledgements}


Hereby I extend my appreciation to my dear colleagues of Biostatistics Group and Cancer Research Center of Shahid Beheshti University of Medical Sciences. This paper is a part of a thesis in Biostatistics Department of paramedical sciences faculty of Shahid Beheshti University of Medical Sciences.

\section{References}

Abdullah NA, Wan Mahiyuddin WR, Muhammad NA, et al (2014). Survival rate of breast cancer patients in Malaysia: a population-based study. Asian Pac J Cancer Prev, 14, 4591-4.

Acil H, Cavdar I (2014). Comparison of quality of life of Turkish breast cancer patients receiving breast conserving surgery or modified radical mastectomy. Asian Pac J Cancer Prev, 15, 5377-81.

Akhtar M, Dasgupta S, Rangwala M (2015). Triple negative breast cancer: an Indian perspective. Breast Cancer : Targets Therapy, 7, 239-43.

Asano J, Hirakawa A, Hamada C, et al (2013). Use of cox's cure model to establish clinical determinants of long-term disease-free survival in neoadjuvant-chemotherapy-treated breast cancer patients without pathologic complete response. Int J Breast Cancer, 354579.

Asif HM, Sultana S, Akhtar N, et al (2014). Prevalence, risk factors and disease knowledge of breast cancer in Pakistan. Asian Pac J Cancer Prev, 15, 4411-6.

Baghestani AR, Shahmirzalou P, Zayeri F, Akbari ME, Hadizadeh M (2015). Prognostic factors for survival in patients with breast cancer referred to omitted cancer research center in Iran. Asian Pac J Cancer Prev, 16, 5081-4.

Bollen L, Wibmer C, Wang M, et al (2015). Molecular phenotype is associated with survival in breast cancer patients with spinal bone metastases. Clin Exp Metastasis, 32, 1-5.

Cancho VG, Rodrigues J, de Castro Mr (2015). A flexible model for survival data with a cure rate: a Bayesian approach. $J$ Applied Statistics, 38, 57-70.

Chen X, Cong Y, Pan L, et al (2014). Luminal (Her2 negative) prognostic index and survival of breast cancer patients. Cancer Epidemiol, 38, 286-90.

Colditz GA, Bohlke K (2014). Priorities for the primary prevention of breast cancer. CA Cancer J Clin, 64, 186-94.

Howell A, Anderson AS, Clarke RB, et al (2014). Risk determination and prevention of breast cancer. Breast Cancer Res, 16, 446.

Kaviani A, Neishaboury M, Mohammadzadeh N, et al (2013). Effects of obesity on presentation of breast cancer, lymph node metastasis and patient survival: a retrospective review. Asian Pac J Cancer Prev, 14, 2225-9.

Kim Y, Yoo KY, Goodman MT (2015). Differences in incidence, mortality and survival of breast cancer by regions and countries in Asia and contributing factors. Asian Pac J Cancer Prev, 16, 2857-70.

Kleinbaum DG, Klein M (2012). Survival analysis: a selflearning text. $3^{\text {rd }}$ ed. New York: Springer, 246.

Lotfnezhad Afshar H, Ahmadi M, Roudbari M, Sadoughi F (2015). Prediction of breast cancer survival through knowledge discovery in databases. Glob J Health Sci, 7, 392-8.

Mahmood H, Faheem M, Mahmood S, et al (2015). Impact of age, tumor size, lymph node metastasis, stage, receptor status and menopausal status on overall survival of breast cancer patients in Pakistan. Asian Pac J Cancer Prev, 16, 1019-24.

Mirzaee M,Azmandian J, Zeraati H, et al (2014). Short-term and long-term survival of kidney allograft: cure model analysis.
Iran J Kidney Dis, 8, 225-30.

Movahedi M, Haghighat S, Khayamzadeh M, et al (2012). Survival rate of breast cancer based on geographical variation in iran, a national study. Iran Red Crescent Med $J, \mathbf{1 4}, 798-804$.

Najafi B, Anvari S, Roshan ZA (2013). Disease free survival among molecular subtypes of early stage breast cancer between 2001 and 2010 in Iran. Asian Pac J Cancer Prev, 14, 5811-6.

Narod SA (2013). Tumour size predicts long-term survival among women with lymph node-positive breast cancer. Current Oncol, 19, 249-53.

Orang E, Marzony ET, Afsharfard A (2013). Predictive role of tumor size in breast cancer with axillary lymph node involvement - can size of primary tumor be used to omit an unnecessary axillary lymph node dissection? Asian Pac J Cancer Prev, 14, 717-22.

Othus M, Barlogie B, Leblanc ML, et al (2012). Cure models as a useful statistical tool for analyzing survival. Clin Cancer Res, 18, 3731-6.

Rahimzadeh M, Baghestani AR, Gohari MR, et al (2014). Estimation of the cure rate in Iranian breast cancer patients. Asian Pac J Cancer Prev, 15, 4839-42.

Rama R, Swaminathan R, Venkatesan P (2010). Cure models for estimating hospital-based breast cancer survival. Asian Pac J Cancer Prev, 11, 387-91.

Sipetic-Grujicic SB, Murtezani ZH, Neskovic-Konstatinovic $\mathrm{ZB}$, et al (2014). Multivariate analysis of prognostic factors in male breast cancer in Serbia. Asian Pac J Cancer Prev, 15, 3233-8.

Taghavi A, Fazeli Z, Vahedi M, et al (2012). Increased trend of breast cancer mortality in Iran. Asian Pac J Cancer Prev, 13, 367-70.

Veisy A, Lotfinejad S, Salehi K, et al (2015). Risk of breast cancer in relation to reproductive factors in North-West of Iran, 2013-2014. Asian Pac J Cancer Prev, 16, 451-5.

Veronesi U, Cascinelli N, Mariani L, et al (2002). Twentyyear follow-up of a randomized study comparing breastconserving surgery with radical mastectomy for early breast cancer. N Engl J Med, 347, 1227-32.

Xing MY, Xu SZ, Shen P (2014). Effect of low-fat diet on breast cancer survival: a meta-analysis. Asian Pac J Cancer Prev, 15, 1141-4.

Zeichner SB, Cavalcante L, Suciu GP, et al (2014). Long-term survival of women with locally advanced breast cancer with $>/=10$ involved lymph nodes at diagnosis. Asian Pac J Cancer Prev, 15, 3435-41.

Ziaei JE, Sanaat Z, Asvadi I, et al (2013). Survival analysis of breast cancer patients in northwest Iran. Asian Pac J Cancer Prev, 14, 39-42. 